\title{
Reviews
}

\section{Neuropeptidergic versus cholinergic and adrenergic regulation of islet hormone secretion}

\author{
B. Ahrén ${ }^{1}$, G. J. Taborsky, Jr. ${ }^{2}$ and D. Porte, Jr². \\ ${ }^{1}$ Departments of Pharmacology and Surgery, Lund. University, Lund, Sweden and ${ }^{2}$ Division of Endocrinology and Metabolism, \\ Department of Medicine, Veterans Administration Medical Center, and the ${ }^{2}$ University of Washington, Seattle, Washington, USA
}

The pancreatic islets are richly supplied with autonomic nerves. The fibres follow the arterioles, penetrate the islets and terminate close to the endocrine cells. This morphological relationship makes it likely that the nerves are involved in the physiological regulation of islet function. A great number of papers have been published describing the effects of electrical stimulation of autonomic nerves and of presumed neurotransmitters and blocking agents on islet function. During the last decade several reviews have summarized these findings and dealt with various aspects of neuroregulation of islet hormone secretion [1-4]. In general, these reviews have attempted to explain the effects of neural activation upon islet function in terms of the known effects of the classical adrenergic and cholinergic neural inputs to the islets. However, recent studies suggest that peptidergic input may also be important. Therefore, in this review we will highlight the new studies of the neuroregulation of insulin, glucagon, somatostatin and pancreatic polypeptide secretion, and specifically emphasize the role of the neuropeptides, in addition to the classical neurotransmitters acetylcholine and noradrenaline, as potential mediators of these effects.

\section{Cholinergic-islet interactions}

\section{Anatomy}

The preganglionic cholinergic nerves which innervate the pancreas originate in the dorsal motor nucleus of the vagus and in the nucleus ambiguus, travel in the vagus nerve and terminate at ganglia within the pancreas, sometimes within the islets [2]. These ganglia integrate neural signals and control the activity of the intrapancreatic postganglionic fibres which innervate individual endocrine cells. Though it was originally believed that all the post-ganglionic fibres within the pancreas were cholinergic, recent evidence suggests that some are peptidergic [5]. The importance of cholinergic innervation for islet function is, however, suggested by the 10-fold higher concentration of choline acetyltranferase in the islets versus the adjacent exocrine tissue [6].

\section{Effects of vagal nerve stimulation and cholinergic agonists and antagonists on islet hormone secretion}

Insulin secretion. Electrical activation of the vagus has been shown to stimulate insulin secretion in several species, including dogs [7], calves [8] and pigs $[9,10]$. The response in dogs and calves is inhibited by atropine [7,8], implicating an interaction of the neurotransmitter acetylcholine with a post-ganglionic muscarinic receptor. However, in pigs atropine does not block the response, suggesting non-cholinergic, perhaps peptidergic, neurotransmission [9]. Neurotransmission at the ganglia is, however, mediated by a classical nicotinic action of acetylcholine, since hexamethonium blocks the response [9].

Pharmacologic stimulation with the cholinergic agonists acetylcholine or carbachol induces insulin secretion both in vitro [11] and in vivo [3,12]. Such stimulation could be due either to activation of nicotinic receptors on the intrapancreatic ganglia or to activation of muscarinic receptors on the B cells themselves. Since atropine blocks many of these responses, it appears that the vagus stimulates insulin secretion by a muscarinic mechanism in most circumstances.

Glucagon secretion. Glucagon is released during electrical stimulation of the vagus in the dog [7], calf [8] and pig $[9,10]$. In the calf, the response is inhibited by atropine [8] but in the pig [9] it is not. Thus, there is evidence for both muscarinic and non-muscarinic, possibly peptidergic, mediation of vagally-induced glucagon release. Exogenous acetylcholine can clearly stimulate glucagon secretion, as shown both in vitro in the isolated perfused dog pancreas [11] and in vivo in the dog and the mouse $[12,13]$. Again, however, it is necessary that atropine block these responses before ascribing them to activation of muscarinic receptors on the A cells. Indeed, the importance of muscarinic mechanisms in the physiological control of glucagon secretion remains in doubt because, in man, the muscarinic agent bethanechol does not influence plasma glucagon levels, and because the glucagon response to insulin-induced hypoglycaemia is not blocked by atropine [14]. These data are consistent 
with the stimulation of glucagon secretion during hypoglycaemia by a peptidergic neurotransmitter, or, more directly, by hypoglycaemia itself or by adrenergic activation.

Somatostatin secretion. The effects of vagal nerve stimulation and cholinergic agonists on pancreatic somatostatin secretion are variable. In perfused pig pancreas in vitro, both vagal nerve activation and acetylcholine inhibit somatostatin output, effects that are blocked by atropine [15]. In perfused canine pancreas, acetylcholine inhibited somatostatin secretion in one study [16] and stimulated somatostatin secretion or had no effect in another study [17]. In vivo acetylcholine stimulated somatostatin secretion from dog pancreas [12]. In a recent study in the dog, vagal nerve stimulation produced a clear but small increase of pancreatic somatostatin secretion that was only partially blocked by high doses of atropine [18]. Hence, vagal nerve stimulation and acetylcholine seem to stimulate pancreatic somatostatin secretion in vivo, at least in the dog. However, since vagally-induced somatostatin secretion is only partially blocked by atropine [18], a non-cholinergic, possibly peptidergic, mediation is also likely. While there are other studies on the effects of vagal stimulation on peripheral or portal venous somatostatin levels, these are not relevant here because the changes of peripheral or portal venous somatostatin reflect gastrointestinal rather than pancreatic D-cell activity [19].

Pancreatic polypeptide (PP) secretion. The importance of vagal, cholinergic mechanisms for PP secretion has been clearly established $[2,20]$. Electrical stimulation of the vagus potently enhances PP secretion in the pig [20], calf [8] and $\operatorname{dog}$ [18]. In the pig and dog, the response is largely inhibited by atropine $[18,20]$, whereas in the calf it is inhibited completely by atropine [8]. Acetylcholine stimulates PP secretion by a muscarinic mechanism [12]. Moreover, vagotomy inhibits the PP response to (a) meals [5], (b) the stimulatory effect of hypoglycaemia and (c) the inhibitory effect of hyperglycaemia [20]. These findings demonstrate that vagal, cholinergic activity is a major determinant of PP secretion. Further, they imply that under carefully controlled conditions, PP secretion may be a useful marker of the cholinergic input to the pancreas.

\section{Possible physiological importance of cholinergic-islet interactions}

While effects of cholinergic stimulation are in general known, a physiologic role for the cholinergic nerves in islet hormone secretion has been demonstrated in only a few instances. One of these is the early insulin response to a meal [4]. This response occurs during the first minutes after ingesting food. It is not related to absorption of nutrients since it occurs prior to any change in plasma glucose levels, and since it occurs in response to either oral saccharin, water or sham feeding [4]. The response is totally inhibited by atropine or vagotomy, which demonstrates its dependence on cholinergic nerves [4]. Interestingly, when the early insulin response to a meal is absent, glucose tolerance is impaired [21]. An early hormone response to feeding has also been demonstrated for PP [5] and glucagon [4]. The early glucagon response, like the early insulin response, is mediated by a cholinergic mechanism [4].

Cholinergic mechanisms may also be involved in the enhanced insulin secretion observed in certain animal models of obesity [4], in the regulation of basal insulin secretion [3], in glucose-induced insulin secretion [2] and in postprandial insulin secretion [22]. However, studies which do not show major effects of cholinergic blockade or vagotomy on insulin secretion under these conditions have also been reported [2]. Therefore, it must be emphasized that the role of cholinergic nerves and the vagus in the control of the insulin secretion deserves further study.

\section{Adrenergic-islet interactions}

\section{Anatomy}

Fluorescence histochemistry and immunohistochemistry have revealed a rich supply of catecholaminergic nerves in the pancreas of several species $[2,3,23]$. Catecholaminergic fibres from the splanchnic nerves pass through the coeliac ganglion and enter the pancreas as part of the mixed autonomic nerve. These fibres are associated with the blood vessels supplying the islets and closely approach individual islet cells $[2,3,23]$. The effects of stimulation of these nerves and of administration of the neurotransmitter noradrenaline have been studied extensively [1-3].

\section{Effects of sympathetic nerve stimulation on islet hormone secretion}

Insulin secretion. Electrical stimulation of the splanchnic nerve, or of the mixed pancreatic nerve after atropine pre-treatment, inhibits the insulin response to glucose in dogs [24] and calves [25]. This inhibition is reversed by the alpha-adrenoceptor antagonist phentolamine [25]. This latter result has usually been interpreted to suggest that noradrenaline mediates the inhibition. However, it has recently been recognized that selective $\alpha$-adrenoceptor blockade results in net $\beta$-adrenoceptor stimulation $[26,27]$ which, by itself, could counteract an inhibition of insulin secretion produced by a non-adrenergic mechanism.

Basal insulin release, like glucose-induced insulin release, is decreased by sympathetic nerve stimulation in several species, including dogs [28]. In pigs, however, basal insulin levels increased during sympathetic nerve stimulation [10]. Recently it was shown that intrapancreatic infusion of noradrenaline could not reproduce 
the inhibitory effect of sympathetic nerve activation on basal insulin release in dogs, suggesting that the inhibition is mediated by a non-adrenergic mechanism [28]. Thus, the role of neuronally released noradrenaline in mediating the inhibition of basal insulin release remains to be conclusively proven.

Glucagon secretion. Sympathetic nerve stimulation increases glucagon secretion in all species examined, but the mechanism appears to vary $[10,24,25,28]$. In the calf, ganglionic blockade, but not combined $\alpha$ - and $\beta$ adrenoceptor blockade, inhibits the glucagon response to splanchnic nerve stimulation, suggesting non-adrenergic, perhaps peptidergic, neurotransmission [25]. In the pig, $\alpha$-adrenoceptor but not $\beta$-adrenoceptor blockade markedly inhibits the glucagon response to splanchnic nerve stimulation [10]. In the dog, local noradrenaline infusion could only partially reproduce the increment of glucagon secretion observed during sympathetic nerve stimulation, suggesting both adrenergic and non-adrenergic mechanisms [28].

Somatostatin and PP secretion. Pancreatic somatostatin secretion is inhibited by splanchnic nerve stimulation both in vitro and in vivo $[15,29,30]$. Alpha-adrenocepter blockade eliminates the inhibition in vitro $[15,29]$; again, the resultant unopposed $\beta$-adrenergic stimulation $[25,27]$ might simply offset the original inhibition even if produced by a non-adrenergic mechanism. Recently, it was shown that intrapancreatic infusions of noradrenaline in dogs cannot reproduce the inhibitory effect of sympathetic nerve stimulation on pancreatic somatostatin secretion [30]. Thus, in vivo the inhibition seems non-adrenergic [30]. PP secretion is increased by splanchnic nerve stimulation in the calf, an effect blocked completely by hexamethonium, partially by phentolamine and not at all by propranolol [31]. In the dog, however, electrical activation of the pancreatic sympathetic nerves produces an inhibition of PP secretion by a mechanism that, again, seems non-adrenergic [30].

\section{Interpretation of adrenergic blocking experiments}

As outlined above, the mechanism of sympathetic neural modulation of islet function has often been inferred from the ability of various receptor antagonists to alter the hormone response to nerve stimulation. While this approach can provide some useful mechanistic information, a cautious interpretation of the resulting data is indicated. This caution results from an increasing recognition that (1) the endogenous catecholamines, noradrenaline and adrenaline, are dual agonists having both $\alpha$ - and $\beta$-adrenergic properties, and (2) adrenergic antagonists can alter not only the action of catecholamines but also their release and clearance. For example, $\alpha$-adrenoceptor antagonists are thought to potentiate noradrenaline release by blocking presynaptic

$\alpha$-adrenoceptors [27]. Alpha-adrenoceptor antagonists may also diminish the uptake of catecholamines, thereby increasing local catecholamine levels [26]. Both actions, coupled with blockade of the $\alpha$-adrenoceptors on islet cells, could produce a $\beta$-adrenoceptor stimulation which offsets, rather than blocks, an inhibition originally produced by a non-adrenergic mechanism. Similarly, $\beta$-adrenoceptor blockade has been suggested to inhibit the clearance of catecholamines [32] and therefore might increase an $\alpha$-adrenoceptor stimulation which, if unrecognized, would lead to an overestimate of the original $\beta$-adrenoceptor contribution to the changes of islet function. In addition, other agents besides adrenergic blockers can affect adrenergic nerve activity. For example, barbiturate anaesthesia is known to inhibit the reflex activation of the adrenergic nerves [33] and might also reduce the amount of noradrenaline released during direct electrical stimulation of these nerves. Since this suppressive effect probably varies with the type and level of anaesthesia, direct comparisons of results from seemingly analogous experiments are difficult. Measurement of pancreatic venous noradrenaline levels might allow such comparisons. With such an index of the degree of adrenergic activation, one could assess the effects of adrenergic blocking agents and anaesthesias and gain a clearer understanding of the mechanism by which these neurally mediated changes of islet function occur. It must be emphasized, however, that noradrenaline may not mediate all the effects of sympathetic nerve stimulation on islet function $[28,30]$. Hence, other potential mediators, such as pancreatic neuropeptides, must also be considered.

\section{Effects of pharmacological $\alpha$ - and $\beta$-adrenoceptor stimulation on islet hormone secretion}

Insulin secretion. There is now a large literature demonstrating the effects of $\alpha$ - and $\beta$-adrenoceptor agonists and antagonists on insulin secretion in a variety of species, including man. It is generally accepted that $\alpha$-adrenoceptor agonism inhibits insulin secretion, and that $\beta$ adrenoceptor agonism enhances insulin release [1-3, 34]. Since the endogenous catecholamines, adrenaline and noradrenaline, have both $\alpha$ - and $\beta$-adrenoceptor properties, their net action on insulin secretion depends on their relative effectiveness to stimulate $\alpha$-vs $\beta$-adrenergic receptors. It also depends on the sensitivity of these receptors. This sensitivity can be altered, for example by hypoxia [35]. Other factors such as the plasma glucose level, which often increases during adrenergic activation, can modify the islet response to catecholamines both by directly stimulating insulin release and by potentiating the effectiveness of a $\beta$-adrenoceptor stimulation. Thus, the net effect may be complex.

Recently, certain pharmacologic studies in other tissues have suggested that both $\alpha$ - and $\beta$-adrenergic receptors might be further subdivided into $\alpha_{1}$ and $\alpha_{2}$ and $\beta_{1}$ and $\beta_{2}$ adrenoceptors, respectively. An $\alpha_{2}$-adreno- 
ceptor subtype seems to be responsible for the inhibition of glucose-induced insulin secretion, whereas the regulation of basal insulin secretion may involve both $\alpha_{1}$ - and $\alpha_{2}$-adrenoceptors [36].

Several studies indicate that the $\beta$-adrenoceptor involved in stimulating insulin secretion is of the $\beta_{2}$ - type $[2,3]$. Thus, studies in dogs and mice have shown that selective $\beta_{2}$-adrenoceptor agonists stimulate insulin secretion, and that selective $\beta_{1}$-adrenoceptor antagonists do not block the insulin release induced by non-selective $\beta$-adrenoceptor stimulation $[3,37]$. In addition, selective $\beta_{1}$-adrenoceptor stimulation fails to induce insulin release in mice [3]. However, a species difference seems to exist since, in the rat, selective $\beta_{1}$-adrenoceptor agonism stimulates insulin secretion, and selective $\beta_{1}$-adrenoceptor antagonism blocks the insulin secretory response to non-selective $\beta$-adrenoceptor stimulation [38]. This species difference emphasizes the limitations of the subclassification of the adrenoceptors.

Glucagon secretion. Glucagon secretion from the perfused dog pancreas has been shown to be stimulated by both $\alpha$ - and $\beta$-adrenoceptor agonists [2]. For example, adrenaline or noradrenaline in the presence of propranolol ( $\alpha$-agonism) increases glucagon secretion; this response is inhibited by phenoxybenzamine [39]; isoprenaline, a $\beta$-adrenoceptor agonist, also stimulates glucagon secretion [37]. The finding that selective $\beta_{2}$-adrenoceptor agonism stimulates glucagon secretion and that selective $\beta_{1}$-adrenoceptor antagonism fails to inhibit isoprenaline-induced glucagon secretion makes it likely that a $\beta_{2}$-receptor-subtype mediates this effect [37].

Somatostatin and PP secretion. Both somatostatin and $P P$ secretion, like insulin secretion, seem to be increased by $\beta$-adrenoceptor stimulation and decreased by $\alpha$ adrenoceptor stimulation. Thus, isoprenaline ( $\beta$-adrenoceptor stimulation) stimulates somatostatin secretion from dog pancreas in vitro [39] and in vivo [19], and adrenaline during propranolol ( $\alpha$-adrenoceptor stimulation) inhibits pancreatic somatostatin secretion from the dog pancreas in vitro [39]. Furthermore, $\beta$-adrenoceptor stimulation (adrenaline + phentolamine) increases PP secretion, and $\alpha$-adrenoceptor stimulation (adrenaline + propranolol) inhibits PP secretion in man [40].

\section{Physiological importance of sympathetic influences on islet function}

The most convincing evidence for a role of the sympathetic nervous system in controlling islet function comes from studies of various stress states. Stress is known to activate both sympathetic nerves and the adrenal medulla, and is usually accompanied by a stimulation of glucagon release and a relative inhibition of insulin release; i.e. a lower insulin level than expected for the concomitant hyperglycaemia. The role of adrenergic activation in mediating the glucagon response to insulin-induced hypoglycaemia is, however, controversial. Certain studies in man have suggested that the hypoglycaemia itself, not the adrenergic activation, is the major stimulus for glucagon secretion [41]. In contrast, more recent studies in isolated perfused rat pancreas have shown that the stimulation of glucagon secretion produced by severe glucopenia might activate adrenergic nerves locally within the pancreas, since the stimulation of glucagon secretion was markedly reduced by combined $\alpha$ - and $\beta$-adrenoceptor blockade [42]. It remains to be critically evaluated whether loss of this local activating ability is responsible for the insensitivity of the A cell to hypoglycaemia in diabetes.

Other types of stresses that affect islet hormone secretion include hypoxia, hypothermia and exercise. Hypoxia directly impairs $\beta$-adrenergic function and produces an exaggerated inhibition of insulin and stimulation of glucagon release by an $\alpha$-adrenergic mechanism [35]. Hypothermia also produces an inhibition of insulin release which is reversed by $\alpha$-adrenoceptor blockade [43]. The physiologic stress of exercise produces an inhibition of insulin release reversed by $\alpha$ adrenoceptor blockade [44]. Exercise also produces an increase of glucagon secretion which is diminished both by $\alpha$-and $\beta$-adrenoceptor blockade [44].

In summary, a variety of stresses produce an inhibition of insulin secretion that is reversed by $\alpha$-adrenoceptor blockade and a stimulation of glucagon secretion that is inhibited by both $\alpha$ - and $\beta$-adrenoceptor blockade. However, for the reason outlined above, that demonstration is not conclusive evidence that catecholamines mediate the original inhibition of insulin secretion. Interpretation of the effects of adrenergic blockade on glucagon secretion are more straightforward, since either $\alpha$ - or $\beta$-adrenoceptor stimulation seems to stimulate glucagon release. Both circulating adrenaline released from the adrenal medulla and local noradrenaline released from adrenergic nerves within the pancreas may participate in these responses.

These is also evidence that the basal adrenergic tonus has a dual effect on basal insulin secretion. Thus, $\alpha$ adrenoceptor blockade increases basal insulin secretion [34], suggesting a tonic $\alpha$-adrenergic inhibition, and $\beta$ adrenoceptor blockade decreases basal insulin secretion [3], suggesting a simultaneous tonic $\beta$-adrenoceptor stimulation. The opposing $\alpha$ - and $\beta$-adrenergic influences make it difficult to determine the net effect of basal adrenergic tonus on basal insulin release.

\section{Neuropeptidergic-islet interactions}

\section{Criteria for pancreatic neuropeptides}

Peptides have long been thought to influence islet function. For example, polypeptide hormones released from the gastrointestional tract are thought to potentiate the 
Table 1. Criteria for pancreatic neuropeptides

Criterion 1: Pancreatic localization

Criterion 2: Pancreatic release during neural activation

Criterion 3: Ruling out classical neurotransmitters

Criterion 4: Effects on islet hormone secretion

Criterion 5: Neural effects inhibited by specific neuropeptide antagonist

release of insulin in response to ingested nutrients [3, 22]. Moreover, certain hypothalamic/pituitary peptides can influence both insulin and glucagon release. For example, vasopressin apparently potentiates the release of glucagon during hemorrhagic stress [45]. In addition, peptides released from islet cells may influence the secretions of other islet cell types in a paracrine manner. Three paracrine interactions within islets have received convincing experimental support. First, selective suppression of pancreatic somatostatin secretion stimulates glucagon secretion [46], suggesting that islet D cells tonically restrain the function of their neighboring A cells. Second, glucagon can stimulate somatostatin secretion, probably in paracrine fashion [47]. Third, endogenous insulin or B-cell activity apparently inhibits glucagon secretion [47]. Although it was originally proposed that this latter B-A cell interaction was paracrine, recent descriptions of islet blood flow going from the B cell core to the D- and A-cell mantle [47] make it more probable that endogenous insulin restrains glucagon secretion by a local, endocrine mechanism. This interpretation was recently supported by the demonstration that high titre insulin antibodies increase glucagon secretion [48]. Analogous experimental approaches with pancreatic somatostatin have usually failed, suggesting that this peptide probably acts via a paracrine route rather than via a local endocrine route. However, the purpose of this review is not to discuss the influence of either hormonal or paracrine peptides, but instead to discuss those peptides which may influence islet function via local release from intrapancreatic nerves.

Many different peptides have been found in nerve terminals throughout the gastrointestinal tract and the brain, where they presumably act as neurotransmitters or neuromodulators. Some of these peptides have also been demonstrated in pancreatic nerves, where they might be regulators of islet function. Although many criteria have been proposed for classical neurotransmitters, five are especially relevant for candidate pancreatic neuropeptides (Table 1). The first criterion is the immunocytochemical demonstration of the peptide in pancreatic nerves. Here a cautious interpretation is indicated, since the antibodies used for such demonstrations have limited recognition sites, and many peptides belong to families which share amino acid sequences. Hence, antibodies may cross-react with other peptides than the peptide to which they were orginally directed. The second criterion is a demonstration that the neuropeptide is actually released from the pancreas during stimulation of the nerve. Third, if the released neuropeptide mediates certain changes of islet function, then complete blockade of the classical adrenergic and cholinergic neurotransmitter actions should not totally prevent the islet changes produced by nerve stimulation. Fourth, administration of the neuropeptide should be able to mimic the remaining islet responses. The fifth and final criterion is to show that blockade of neuropeptide action abolishes the islet response to nerve stimulation. The data relevant to each of the first four criteria will here be given for the candidate pancreatic neuropeptides listed below. Fulfillment of the last criterion awaits development of potent and selective neuropeptide antagonists, which, at present, is just beginning.

\section{Criterion 1: Pancreatic localization}

Vasoactive intestinal polypeptide (VIP). VIP-immunoreactivity has been demonstrated in the fibres supplying pancreatic ganglia and blood vessels in several species, including man, dogs, cats and rats $[5,49,50]$. In humans [50] and dogs [5], these nerves form a peri-insular network, and some fibres even penetrate the islets and terminate on individual endocrine cells. In man this neural substance is chromatographically identical to porcine VIP [50]. Since pancreatic denervation in the dog does not alter VIP immunostaining in the pancreas, it is suggested that VIP nerves are intrinsic to the pancreas [5]. Thus, preganglionic fibres entering the pancreas apparently control the activity of the post-ganglionic, VIPergic fibres within.

Cholecystokinin (CCK). Nerve fibres containing CCKlike immunoreactivity innervate the ganglia and pancreatic islets of cats, pigs and hamsters $[49,51]$. Since no CCK nerve cell bodies seem to exist within the pancreas, it has been argued that the CCK nerves are extrinsic, postganglionic fibres [51]. Several different forms of CCK exist having 4, 8, 12, 33, 39 or 58 amino acids; however, it has been suggested that the neural CCK-like immunoreactivity is a short form, mainly CCK-4 [51]. A physiologic role for CCK in islet function is suggested by recent observations of CCK receptors on islet cells [52].

Gastrin releasing polypeptide (GRP). There is recent evidence for a third peptidergic nerve population in the pancreas of several species that binds antibody recognizing GRP, a 27 amino acid polypeptide resembling bombesin [53]. Bombesin-like immunoreactivity has also been demonstrated in porcine pancreatic ganglia [53].

Galanin. Recently it was demonstrated that galanin-like immunoreactivity occurs in nerve fibres in association with dog islets, making it likely that this 29 amino acid peptide is an intrapancreatic neuropeptide [54]. 
Neuropeptide $Y(N P Y)$. It has also recently been shown that some nerves within the pancreas react with antibodies to NPY-like immunoreactivity [23]. The NPY nerves seem to innervate both vessels and endocrine cells, and some of the NPY nerves, but not all, contain noradrenaline. This leads to the suggestion that this 36 amino acid peptide is a sympathetic neurotransmitter. More recent evidence suggests, however, that this classification is an oversimplification of the physiologic role of NPY.

Calcitonin gene-related peptide (CGRP). CGRP-nerves, originally found in the intestine, thyroid and brain, have now been identified in the pancreas [55]. These nerves are scattered throughout the pancreatic tissue of the rat and the mouse and are mainly associated with blood vessels, but some fibres also penetrate the islets [55]. Recent evidence that capsaicin reduces CGRP-staining in the gut suggests that this 37 amino acid neuropeptide might be a sensory neurotransmitter, perhaps also in the pancreas.

Substance $P$ and enkephalin. Other peptides have also been found in the neural elements of gut and pancreas. Thus, ganglia from cat pancreas contain both substance P- and leu-enkephalin-like immunoreactivity [49]. However, species differences seem to exist in that no substance P- or enkephalin-like immunoreacitivity were found in dog pancreas [5].

Summary. The currently available data suggest that VIP, CCK, GRP, galanin, NPY, CGRP, substance P and leu-enkephalin-like immunoreactivity are present in certain intrapancreatic nerves. Given the recent nature of these observations and the current interest in neuropeptides, one can predict that other pancreatic neuropeptides will be discovered soon.

\section{Criterion 2: pancreatic release during neural activation}

It has recently been demonstrated that VIP and GRP are released into the effluent of an isolated, perfused pancreas following electrical stimulation of the attached vagal fibres [56, 57]. It remains, however, to be demonstrated that any of the other candidate neuropeptides listed above are released from the pancreas after neural stimulation. Other studies have demonstrated that nerve stimulation releases certain neuropeptides from various areas of the gut. However, with the exception of VIP and GRP, direct demonstration of such release from pancreatic nerves is still needed.

\section{Criterion 3: ruling out classical neurotransmitters}

Vagally-induced insulin secretion. Vagal nerve stimulation in the pig causes an insulin release which is not affected by atropine but which is totally abolished by hexamethonium [9]. This suggests that preganglionic, cholinergic fibres activate postganglionic, non-cholinergic (perhaps peptidergic), fibres which in turn stimulate insulin secretion. However, atropine markedly diminishes the vagal stimulation of insulin secretion in the dog [7], suggesting that, in this species, acetylcholine is the main post-ganglionic neurotransmitter.

Vagally-induced glucagon secretion. Recent studies in the pig [9] and dog [7] have shown that vagally-induced glucagon secretion is not diminished by a large dose of atropine. Since hexamethonium abolished these responses, peptidergic neurotransmission at the post-ganglionic level has been suggested.

Vagally-induced somatostatin and PP secretion. There is a large increase of PP secretion and a small increase of somatostatin secretion during vagal nerve stimulation in vivo $[18,20]$. Although atropine markedly diminishes both responses, it does not abolish them as hexamethonium does. A pancreatic neuropeptide may thus mediate the residual somatostatin and PP secretion.

Sympathetically-induced changes in islet hormone secretion. The glucagon response to splanchnic nerve stimulation in calves is not inhibited by combined $\alpha$ - and $\beta$ adrenoceptor blockade [25]. The available evidence suggests that GRP mediates this response, since GRP is released by splanchnic nerve stimulation in calves [31], and since exogenous GRP stimulates glucagon release in this species [58].

A recent study which compared the effects of sympathetic nerve stimulation to those of pancreatic noradrenaline infusion in vivo concluded that noradrenaline is probably not the mediator of the sympathetically-induced inhibition of insulin, somatostatin or PP release $[28,30]$; other neurotransmitters, perhaps neuropeptides, may be involved in these responses.

\section{Criterion 4: Effects on islet hormone secretion}

$V I P$. In vivo VIP stimulates insulin and glucagon secretion in several species, including rats [59] and mice [3, 13]. In addition, VIP potentiates glucose-induced insulin release and arginine-induced glucagon release [3, 59]. In vitro VIP stimulates insulin, glucagon and somatostatin secretion from the perfused dog pancreas [60].

CCK. CCK stimulates insulin secretion in several species. In mice $[3,61]$ and in perfused dog pancreas [62], CCK-8 seems to be the shortest CCK variant that is active on insulin secretion; in perfused pig pancreas, the shortest active form is CCK-4 [51]. PP and somatostatin are also released by CCK-4 and CCK- 8 in vivo in the dog $[19,63]$, whereas the effect on glucagon secretion remains to be established. The larger molecular forms of $\mathrm{CCK}$ affect islet function as well [3,61], but this finding is probably not relevant for the neuropeptidergic con- 
cept since the CCK-like immunoreactivity found in pancreatic nerves is probably a shorter form of CCK [51].

GRP. GRP stimulates the secretion of insulin, glucagon and PP as shown in studies in dogs [64] and calves [58].

Galanin. Galanin potently inhibits insulin secretion, potently inhibits somatostatin secretion, stimulates glucagon secretion and produces a marked hyperglycaemia in dogs [54].

NPY. NPY inhibits glucose-induced insulin secretion in mice [23], perfused rat pancreas, and from isolated rat islets [65]. The possible influence of NPY on the secretion of the other islet hormones remains to be studied.

CGRP. CGRP inhibits basal and stimulated insulin secretion in mice [55], but the possible effects of CGRP on the secretion of glucagon, somatostatin and PP are unkown.

Substance P. Substance P stimulates insulin and glucagon secretion from perfused dog pancreas in vitro [60], whereas glucose-induced insulin secretion is inhibited in mice in vivo [3]. Substance $P$ has also been shown to stimulate somatostatin secretion from the perfused dog pancreas [60]. The effects of substance $P$ on somatostatin or PP secretion in vivo are not known.

Enkephalin. Certain opioid peptides have both inhibitory and stimulatory effects on islet hormone secretion, depending on the dose, the animal species studied and the conditions of the experiment. For example, in isolated rat islets $\alpha$-endorphin, dynorphin, met-enkephalin and an enkephalin analogue (DAMME) inhibit insulin secretion at high doses and stimulate it at low doses [66]. However, $\beta$-endorphin and leu-enkephalin do not show these dual effects; they stimulate insulin secretion from rat islets over a wide concentration range [66]. Attempts to demonstrate a tonic effect of endogenous opioid peptides on insulin secretion have produced an equally confusing picture: opioid receptor blockade by naloxone transiently depresses basal insulin levels but potentiates the insulin secretory response to glucose in mice [67]. The significance of these opioid actions on islet hormone secretion is not known; it must be recalled that, currently, only leu-enkephalin-like immunoreactivity has been demonstrated in neural structures in pancreas, and then only in the ganglia [49].

Summary. In summary, all the neuropeptides identified in intrapancreatic nerves can influence islet hormone secretion under certain experimental conditions. In general, VIP, CCK and GRP seem to be stimulatory neuropeptides; galanin, NPY and CGRP seem to be inhibitory neuropeptides; and substance $\mathrm{P}$ and met-enkephalin seem to exert more variable effects. The physio- logic relevance of these islet actions remains to be established.

\section{Coexistence of neurotransmitters}

Indirect evidence for peptidergic mediation of neurallyinduced changes of islet function has been presented by citing those instances in which classical adrenergic, muscarinic or nicotinic blockade has failed to abolish the islet responses (see above, criterion 3). This approach is, however, conservative and intentionally ignores potential interactions and synergisms between the newly discorvered neuropeptides and the classical autonomic neurotransmitters. The following section lists the preliminary evidence for such interactions.

First, there is evidence for the existence of more than one neurotransmitter in a single neuron. Thus, VIP is a constituent of many cholinergic nerves [68] and NPY is found in many adrenergic nerves [23]. These neuropeptides are presumably released concomitantly with acetylcholine or noradrenaline, respectively, when the nerves are activated. If so, these peptides are in a position to modulate the action of the classical adrenergic and cholinergic neurotransmitters. Indeed, in salivary glands, VIP is without effect by itself, yet it potentiates the salivary response to acetylcholine, probably by enhancing muscarinic receptor binding of acetylcholine [68]. A similar synergism between VIP and the cholinergic agonist carbachol has been demonstrated for glucagon secretion [13]. In addition to influencing classical neurotransmitter action, neuropeptides may also alter classical neurotransmitter release. For example, NPY can inhibit neuronal noradrenaline release [69]. If modulation of classical neurotransmitter action and release is a general characteristic of neuropeptides, then it will be important to understand how they modulate such responses, when they exert these actions and what role such modulators play in determining the islet responses to the neural input.

\section{The possible role of cholinergic, adrenergic and peptidergic regulation of islet function}

\section{Cholinergic}

Current evidence suggests that the main function of the cholinergic innervation is to enhance meal-related insulin secretion. The strongest evidence for such a role is the cholinergic, vagal mediation of the cephalic phase of insulin release. This anticipatory response primes metabolic pathways for the incoming nutrients and thus promotes their efficient utilization and storage. The role of the cholinergic nervous system in enhancing the islet response to the direct stimulatory effect of absorbed nutrients is less clear, although some evidence exists in the literature. Such an effect is plausible, but has been difficult to prove experimentally because cholinergic block- 
ade and stimulation have major effects on gastrointestinal and exocrine pancreatic function which in turn alter the pattern of direct, nutrient induced stimulation of islet hormone secretion. Finally, there are suggestions that the cholinergic tonus to the islets is chronically increased in obesity, which is associated with hyperinsulinaemia. Thus, vagal stimulation of insulin release may be advantageous in normal individuals because it promotes the efficient storage of ingested nutrients. However, it may also be disadvantageous in obese individuals for the same reason. The physiologic role for the cholinergic nerves on the secretion of glucagon, somatostatin and pancreatic polypeptide is less clear. It seems, however, that the major effect of the cholinergic nerves on the islets is to stimulate the secretion of all four islet hormones.

\section{Adrenergic}

The role of the adrenergic system appears to be predominantly the mediation of the islet responses to stress. Acute stress usually elevates plasma glucose concentration as a protective mechanism for the brain, i.e. to maintain adequate glucose delivery to the central nervous system. The insulin level is kept low to spare glucose utilization by insulin sensitive tissues and to maximize glucose utilization by the insulin independent, neural tissue. The glucagon level is kept high to stimulate glucose production. Adrenergic effects on the islets help to achieve both goals by inhibiting insulin and stimulating glucagon secretion. In contrast, during chronic stresses, e.g. after burns or surgery, where increased delivery of substrate to injured tissue is desired without hyperglycaemia, the catecholamines play a lesser role. Such stress is characterized by an increase in fuel delivery to insulin sensitive tissues. In these stresses, insulin secretion may be only mildly impaired and glucose uptake maximized. Thus, the function of the adrenergic input to the islets is to decrease insulin and to increase glucagon secretion in order to produce marked hyperglycaemia. This hyperglycaemia presumably insures adequate glucose delivery to the brain during periods of acute stress.

\section{Neuropeptidergic}

What then is the role of the neuropeptides in islet function? Candidate neuropeptides are being reported with increasing frequency; at this point it is clear that exogenous neuropeptides can effect islet function, and that some of these are present in islet nerves. Their role as neurotransmitters seem assured because there are a number of islet responses, both to vagal and sympathetic nerve activation, that cannot be explained by either an adrenergic or cholinergic transmitter action. Further, neural activation does release at least certain of these neuropeptides (VIP and GRP) from the pancreas. Hence, some of the criteria for the intrapancreatic neu- ropeptides are fulfilled. However, the definite experiments which will prove the physiologic role of neuropeptides requires blockers of peptide action. Such antagonists are just beginning to be developed; if they turn out to be specific, strong evidence for or against neuropeptidergic involvement in islet hormone secretion will be presented. In the meantime, what can we say about the potential physiologic importance of pancreatic neuropeptides? It is difficult to generalize when discussing their potential physiological role, because the individual neuropeptides have qualitatively different effects on islet hormones secretion. Some, however, like VIP, GRP and CCK stimulate both insulin and glucagon secretion simultaneously. Since insulin and glucagon regulate carbohydrate metabolism in a reciprocal fashion, this stimultaneous stimulation may appear confusing or even futile. In fact, it is the reciprocal nature of insulin and glucagon action that makes adrenergic inhibition of insulin and stimulation of glucagon secretion so potent a hyperglycaemic stimulus. Therefore, we need to examine the consequences of parallel regulation of these two hormones. If insulin and glucagon are simultaneously suppressed or increased, we would expect minimal changes of plasma glucose level but significant changes in glucose turnover. Thus, we might look for circumstances where there is a need for increasing glucose turnover without a need for hyperglycaemia. Increased demand for fuel, e.g. during exercise, might be a situation in which accelerated glucose turnover is needed. The peptidergic activation that might increase both glucagon and insulin secretion would also be expected to maintain hepatic glucose production at or above fasting levels while increasing glucose transport into muscle. In support of this idea there is some evidence for a glucagon response during exercise which is not sensitive to adrenergic blockade [44]. Likewise, certain studies suggest that the glucagon response to less severe hypoglycaemia is also not mediated by adrenergic mechanisms [41], suggesting either a non-adrenergic (peptidergic) component, or reduntant stimulation by hypoglycaemia, adrenergic, cholinergic and peptidergic inputs. Evidence for such reduntant mechanisms has been clearly established for the recovery from hypoglycaemia: the glucagon and epinephrine responses to hypoglycaemia are each nearly sufficient to produce full recovery, yet deficiency of both prevents recovery [70]. By analogy, neuropeptidergic activation might participate with other neural and direct mechanisms in mediating the glucagon as well as the insulin response. However, convincing demonstration of such redundancy will require the same careful experimental design employed in the studies of the recovery from hypoglycaemia.

In summary, the scepticism about neural control of the islets that was present just a few years ago has been replaced by increasing awareness of the importance of neural control of islet function under specific physiologic conditions. The cholinergic nervous system ap- 
pears important for the control of islet function before and during feeding and may also be involved in the pathophysiology of insulin secretion in obesity. The sympathetic nervous system is clearly important for controlling islet function during stress. The role of the local adrenergic nerves of the pancreas in mediating changes of islet function is less clear, but is an area of active investigation. The role of the neuropeptides is just beginning to emerge and promises to lead to new ideas about neural control of the endocrine pancreas.

Acknowledgements. The authors thank Ms. L. Parry, Mr. W. Hintz, Ms. A. Bartlett and Ms. B. Heim for their secretarial help and our colleagues for review of the manuscript. This work was supported by NIH grants AM 12829 and AM 17047, the Research Service of the Veterans Administration and the Swedish Medical Research Council grant 14P-6647. To reduce the length of this review, the number of references has been lowered to the maximum allowed. The authors apologize to all those authors who have contributed significantly to the development of our understanding of neural-islet interactions and have not been cited in this review.

\section{References}

1. Woods SC, Porte Jr D (1974) Neural control of the endocrine pancreas. Physiol Rev 54: 596-619

2. Miller RE (1981) Pancreatic neuroendocrinology: Peripheral neural mechanisms in the regulation of the islets of Langerhans. Endocrin Rev 4: 471-494

3. Ahrén B (1981) On the peptidergic and aminergic regulation of insulin secretion. PhD Thesis, University of Lund, Sweden

4. Steffens AB, Strubbe JH (1983) CNS regulation of glucagon secretion. In: Szabo AJ (ed) Advances in Metabolic Disorders, volume 10. Academic, New York London, pp 221-257

5. Prinz RA, El Sabbagh H, Adrian TE, Bloom SR, Gardner I, Polak JM, Inokuchi H, Bishop AE, Welbourn RB (1983) Neural regulation of pancreatic polypeptide release. Surgery 94: 1011-1018

6. Godfrey DA, Matschinsky FM (1975) Enzymes of the cholinergic system in islets of Langerhans. J Histochem Cytochem 23: 645-651

7. Ahrén B, Taborsky Jr GJ (1986) The mechanism of vagal nerve stimulation of glucagon and insulin secretion in the dog. Endocrinology 118: 1551-1557

8. Bloom SR, Edwards AV (1981) Pancreatic endocrine responses to stimulation of the peripheral ends of the vagus nerves in conscious calves. J Physiol 315: 31-41

9. Holst JJ, Gronholt R, Schaffalitzky de Muckadell OB, Fahrenkrug J (1981) Nervous control of pancreatic endocrine secretion in pigs. 2. The effect of pharmacological blocking agents on the response to vagal stimulation. Acta Physiol Scand 111:9-14

10. Holst JJ, Gronholt R, Schaffalitzky de Muckadell OB, Fahrenkrug $J$ (1981) Nervous control of pancreatic endocrine secretion in pigs. V. Influence of the sympathetic nervous system on the pancreatic secretion of insulin and glucagon, and on the insulin and glucagon response to vagal stimulation. Acta Physiol Scand 113:278-283

11. Iversen $J$ (1973) Effect of acetylcholine on the secretion of glucagon and insulin from the isolated, perfused canine pancreas. Diabetes $22: 381-387$

12. Kaneto A, Sata H, Kaneko T, Yanaihara C, Yanaihara N, Kosaka K (1981) Stimulation of somatostatin, pancreatic polypeptide, glucagon and insulin secretion by acetylcholine infused intrapancreatically in dogs. Biomed Res 2: 57-66

13. Ahrén B, Lundquist I (1982) Interaction of vasoactive intestinal peptide (VIP) with cholinergic stimulation of glucagon secretion. Experientia 38: 405-406

14. Palmer JP, Werner PL, Hollander B, Ensinck JW (1979) Evalua- tion of the control of glucagon secretion by the parasympathetic nervous system in man. Metabolism 28: 549-552

15. Holst JJ, Jensen SL, Knuhtsen S, Nielsen OV (1983) Autonomic nervous control of pancreatic somatostatin secretion. Am J Physiol 245: E542-E548

16. Samols E, Stagner JI (1981) Autonomic function and control of pancreatic somatostatin. Diabetologia 20: 388-392

17. Hermansen K (1980) Secretion of somatostatin from the normal and diabetic pancreas. Studies in vitro. Diabetologia 19:492-504

18. Ahrén B, Paquette TL, Taborsky Jr GJ (1986) Effect and mechanism of vagal nerve stimulation on somatostatin secretion in dogs. Am J Physiol 250: E212-E217

19. Taborsky Jr GJ, Ensinck JW (1984) Contribution of the pancreas to circulating somatostatin-like immunoreactivity in the normal dogs. J Clin Invest $73: 216-223$

20. Schwartz TW, Holst JJ, Fahrenkrug J, Jensen SL, Nielsen OV, Rehfeld JF, Schaffaliltzky de Muckadell OB, Stadil F (1978) Vagal cholinergic regulation of pancreatic polypeptide secretion. J Clin Invest 61: 781-789

21. Stubbe JH (1982) Effects of pancreas transplantation on insulin secretion in rats during ingestion of varying glucose loads. Diabetologia $22: 354-357$

22. Berthoud MR (1984) The relative contribution of the nervous system, hormones, and metabolites to the total insulin response during a meal in the rat. Metabolism 33:18-25

23. Pettersson M, Ahrén B, Lundquist I, Böttcher G, Sundler F (1986) Neuropeptide Y: intrapancreatic neuronal localization and inhibitory effects on glucose-stimulated insulin secretion in the mouse. Cell Tissue Res (in press)

24. Marliss EB, Girardier L, Seydoux J, Wolheim CD, Kanazawa $Y$, Orci L, Renold AE, Porte Jr D (1973) Glucagon release induced by pancreatic nerve stimulation in the dog. J Clin Invest 52: 1246-1259

25. Bloom SR, Edwards AV (1978) Certain pharmacological characteristics of the release of pancreatic glucagon in response to stimulation of the splanchnic nerves. J Physiol 280: 25-35

26. Iversen LL (1971) Role of transmitter uptake mechanisms in synaptic neurotransmission. Br J Pharmacol 41: 571-591

27. Westfall TC (1984) Evidence that noradrenergic transmitter release is regulated by presynaptic receptors. Fed Proc 43: 1352-1357

28. Ahrén B, Veith RC, Taborsky Jr GJ Sympathetic nerve stimulation versus pancreatic norepinephrine infusion in the dog: 1) Effects on basal release of insulin and glucagon. Endocrinology (in press)

29. Roy MW, Lee KC, Jones MS, Miller RE (1984) Neural control of pancreatic insulin and somatostatin secretion. Endocrinology 115 : $770-775$

30. Ahrén B, Veith RC, Paquette TL, Taborsky Jt GJ Sympathetic nerve stimulation versus pancreatic norepinephrine infusion in the dog: 2) Effects on basal release of somatostatin and pancreatic polypeptide. Endocrinology (in press)

31. Bloom SR, Edwards AV (1984) Characteristics of the neuroendocrine responses to stimulation of the splanchnic nerves in bursts in the conscious calf. J Physiol 346: 533-545

32. Cryer PE, Rizza RA, Haymond MW, Gerich JE (1980) Epinephrine and norepinephrine are cleared through $\beta$-adrenergic, but not $\alpha$-adrenergic, mechanisms in man. Metabolism 29 (Suppl 1): $1114-1118$

33. Taborsky Jr GJ, Halter JB, Baum D, Best JD, Porte Jr D (1984) Pentobarbital anesthesia suppresses basal and 2-deoxy-glucose stimulated plasma catecholamines. Am J Physiol 247: R905-R910

34. Ahrén B, Lundquist I (1985) Effects of $\alpha$-adrenoceptor blockade by phentolamine on basal and stimulated insulin secretion in the mouse. Acta Physiol Scand 125: 211-217

35. Baum D, Porte Jr D (1980) Stress hyperglycemia and the adrenergic regulation of pancreatic hormones in hypoxia. Metabolism 29 (Suppl 1): $1170-1185$

36. Skoglund G, Lundquist I, Ahrén B (1986) Effects of $\alpha_{1}$ - and $\alpha_{2}$ adrenoceptor stimulation and blockade on plasma insulin levels in the mouse. Pancreas: $415-420$ 
37. Kaneto A, Miki E, Kosaka K (1975) Effect of $\beta$ - and $\beta_{2}$-adrenoceptor stimulants infused intrapancreatically on glucagon and insulin secretion. Endocrinology 97: 1166-1173

38. Furman BL, Tayo FM (1974) Effect of some $\beta$-adrenoceptor blocking drugs on insulin secretion in the rat. J Pharm Pharmacol 26: $512-517$

39. Samols E, Weir GC (1979) Adrenergic modulation of pancreatic $\mathrm{A}, \mathrm{B}$ and $\mathrm{D}$ cells. $\alpha$-Adrenergic suppression and $\beta$-adrenergic stimulation of somatostatin secretion, $\alpha$-adrenergic stimulation of glucagon secretion in the perfused dog pancreas. J Clin Invest 63: $230-238$

40. Sive AA, Vinik AI, Levitt N (1980) Adrenergic modulation of human pancreatic polypeptide (hPP) release. Gastroenterology 79: 665-672

41. Palmer JP, Henry DP, Benson JW, Johnson DG, Ensinck JW (1976) Glucagon response to hypoglycemia in sympathectomized man. J Clin Invest 57:522-525

42. Hisatomi A, Maruyama H, Orci L, Vasko M, Unger RH (1985) Adrenergically mediated intrapancreatic control of the glucagon response to glucopenia in the isolated rat pancreas. J Clin Invest 75: $420 \sim 426$

43. Helman A, Gilbert M, Pfisler-Lemaire N, Reach G, Assan R (1984) Glucagon and insulin secretion and their biological activities in hypothermic rats. Endocrinology 115: 1722-1728

44. Brockman RP, Halvorson R (1982) Glucose, glucagon, and insulin during adrenergic blockade in exercising sheep. J Appl Physiol 52: 315-319

45. Dunning BE, Verbalis JG, Fawcett CP (1985) Evidence for participation of the neurohypophysial hormones in the hyperglucagonemic response to hemorrhage in the rat. Neuroendocrinology 41 : 385-389

46. Klaff LJ, Taborsky Jr GJ Pancreatic somatostatin as a mediator of the inhibition of glucagon by hyperglycemia. Diabetes (in press)

47. Samols E, Bonner-Weir S, Weir GC (1986) Intra-islet insulinglucagon-somatostatin relationships. Clin Endocrinol Metab 14: $33-58$

48. Maruyama H, Hisatomi A, Orci L, Grodsky GM, Orci RH (1984) Insulin within islets is a physiologic glucagon release inhibitor. $\mathrm{J}$ Clin Invest 74: 2296-2299

49. Larsson LI (1979) Innervation of the pancreas by substance $P$, enkephalin, vasoactive intestinal polypeptide and gastrin-CCK immunoreactive nerves. J Histochem Cytochem 27: 1283-1284

50. Bishop AE, Polak JM, Green IC, Bryant MG, Bloom SR (1980) The location of VIP in the pancreas of man and rat. Diabetologia 18: $73-78$

51. Rehfeld JF, Larsson LI, Goltermann NR, Schwartz TW, Holst JJ, Jensen SL, Morley JS (1980) Neural regulation of pancreatic hormone secretion by the C-terminal tetrapeptide of CCK. Nature 284: $33-38$

52. Verspohl EJ, Ammon HPT, Williams JA, Goldfine ID (1986) Evidence that cholecystokinin interacts with specific receptors and regulates insulin release in isolated rat islets of Langerhans. Diabetes $35: 38-43$

53. Moghimzadeh E, Ekman R, Håkanson R, Yanaihara N, Sundler F (1983) Neuronal gastrin-releasing peptide in the mammalian gut and pancreas. Neuroscience 10: 553-563

54. Dunning BE, Ahrén B, Veith RC, Böttcher G, Sundler F, Taborsky Jr GJ (1986) Galanin: A novel pancreatic neuropeptide. Am J Physiol 251: E127-E133

55. Pettersson M, Ahrén B, Böttcher G, Sundler F (1986) Calcitonin gene-related peptide: Occurrence in pancreatic islets in the mouse and the rat and inhibition of insulin secretion in the mouse. Endocrinology 119: 865-869

56. Holst JJ, Fahrenkrug J, Knuhtsen S, Jensen SL, Poulsen SS, Nielsen OV (1984) Vasoactive intestinal polypeptide (VIP) in the pig pancreas: Role of VIPergic fibers in control of fluid and bicarbonate secretion. Regul Pept 8: 245-249

57. Knuhtsen S, Holst JJ, Jensen SL, Nielsen OV (1985) Gastrin releasing peptide: Effect on exocrine secretion and release from isolated perfused pig pancreas. Am J Physiol 248: G281-G287

58. Bloom SR, Edwards AV (1983) Endocrine responses to exogenous bombesin and gastrin releasing peptide in conscious calves. J Physiol 344: 37-48

59. Szecówka J, Lins PE, Tatemoto K, Efendić S (1983) Effects of porcine intestinal heptacosapeptide and vasoactive intestinal polypeptide on insulin and glucagon secretion in rats. Endocrinology 112: $1469-1473$

60. Hermansen K (1980) Effects of substance P and other peptides on the release of somatostatin, insulin and glucagon in vitro. Endocrinology 107: 256-261

61. Ahrén B, Hedner P, Lundquist I (1986) Effects of six cholecystokinin $(\mathrm{CCK})$ fragments on insulin secretion in the mouse. Acta Pharmacol Toxicol 58:115-120

62. Hermansen K (1984) Effects of cholecystokinin (CCK)-4, nonsulfated CCK -8 and sulfated CCK-8 on pancreatic somatostatin, insulin and glucagon secretion in the dog: Studies in vitro. Endocrinology 114: 1770-1775

63. Beglinger C, Taylor IL, Grossman MI, Solomon TE (1984) Pancreatic polypeptide release: Role of stimulants of exocrine pancreatic secretion in dogs. Gastroenterology 87:530-536

64. McDonald TJ, Ghatei MA, Bloom SR, Adrian TE, Mochizaki T, Yanaihara C, Yanaihara N (1983) Dose-response comparisons of canine plasma gastroenteropancreatic hormone responses to bombesin and the porcine gastrinreleasing peptide (GRP). Regul Pept 5: 125-137

65. Moltz JH, McDonald JK (1985) Neuropeptide Y: Direct and indirect action on insulin secretion in the rat. Peptides 6:1155-1159

66. Green IC, Ray K, Pervin D (1983) Opioid peptide effects on insulin release and cAMP in islets of Langerhans. Horm Metab Res $15: 124-128$

67. Ahrén B, Lundquist I (1984) Effects of naloxone on basal and stimulated insulin secretion in the mouse. Eur J Pharmacol 102 : 135-139

68. Lundberg JM, Anggård A, Fahrenkrug J, Hökfelt T, Mutt V (1980) Vasoactive intestinal polypeptide in cholinergic neurons of exocrine glands: functional significance of coexisting transmitters for vasodilatation and secretion. Proc Natl Acad Sci USA 77: $1651-1655$

69. Lundberg JM, Stjärne L (1984) Neuropeptide Y (NPY) depresses the secretion of ${ }^{3} \mathrm{H}$-noradrenaline and the contractile response evoked by field stimulation in rat vas deferens. Acta Physiol Scand 120: 477-479

70. Gerich J, Cryer P, Rizza R (1980) Hormonal mechanisms in acute glucose counterregulation: The relative roles of glucagon, epinephrine, norepinephrine, growth hormone, and cortisol. Metabolism 29 (Suppl 1): 1164-1175

Dr. Bo Ahrén

Department of Pharmacology

Sölvegatan 10

S-22362 Lund

Sweden 